\title{
Fine structure of modal focusing effect in a three dimensional plasma-sheath-lens
} formed by disk electrodes

\author{
Stamate, Eugen; Yamaguchi, Masahito
}

\section{Published in:}

Applied Physics Letters

Link to article, DOI:

$10.1063 / 1.4930063$

Publication date:

2015

Document Version

Publisher's PDF, also known as Version of record

Link back to DTU Orbit

Citation (APA):

Stamate, E., \& Yamaguchi, M. (2015). Fine structure of modal focusing effect in a three dimensional plasmasheath-lens formed by disk electrodes. Applied Physics Letters, 107(9), [094106].

https://doi.org/10.1063/1.4930063

\section{General rights}

Copyright and moral rights for the publications made accessible in the public portal are retained by the authors and/or other copyright owners and it is a condition of accessing publications that users recognise and abide by the legal requirements associated with these rights.

- Users may download and print one copy of any publication from the public portal for the purpose of private study or research.

- You may not further distribute the material or use it for any profit-making activity or commercial gain

- You may freely distribute the URL identifying the publication in the public portal 


\title{
Fine structure of modal focusing effect in a three dimensional plasma-sheath-lens formed by disk electrodes
}

\author{
Eugen Stamate ${ }^{1,2, a)}$ and Masahito Yamaguchi ${ }^{3,2, b)}$ \\ ${ }^{1}$ Department of Energy Conversion and Storage, Technical University of Denmark, Frederiksborgvej 399, \\ Roskilde 4000, Denmark \\ ${ }^{2}$ Venture Business Laboratory, Nagoya University, C3-1, Chikusa-ku, Nagoya 464-8603, Japan \\ ${ }^{3}$ Department of Electrical Engineering and Computer Science, Nagoya University, C3-1, Chikusa-ku, \\ Nagoya 464-8603, Japan
}

(Received 15 June 2015; accepted 22 August 2015; published online 1 September 2015)

\begin{abstract}
Modal and discrete focusing effects associated with three-dimensional plasma-sheath-lenses show promising potential for applications in ion beam extraction, mass spectrometry, plasma diagnostics and for basic studies of plasma sheath. The ion focusing properties can be adjusted by controlling the geometrical structure of the plasma-sheath-lens and plasma parameters. The positive and negative ion kinetics within the plasma-sheath-lens are investigated both experimentally and theoretically and a modal focusing ring is identified on the surface of disk electrodes. The focusing ring is very sensitive to the sheath thickness and can be used to monitor very small changes in plasma parameters. Three dimensional simulations are found to be in very good agreement with experiments.
\end{abstract} (C) 2015 AIP Publishing LLC. [http://dx.doi.org/10.1063/1.4930063]

The sheath is a space charge layer interfacing the quasineutral plasma with the walls defining the plasma volume or with immersed objects. ${ }^{1}$ Besides its essential role in controlling the charge loss to sustain the discharge, the sheath is one of the main plasma aspects used to control the ion and electron fluxes. For example, some of the most successful applications of plasma processing, including reactive ion etching, ${ }^{2}$ physical vapor deposition by magnetron sputtering, ${ }^{3}$ ion beam extraction, ${ }^{4}$ and plasma immersion ion implantation $^{5}$ are based on ions accelerated in a sheath that are precisely tuned in term of energy, flux, and incidence angle. Process uniformity is one of the key parameters, so that ions are usually accelerated perpendicular to the surface or the extraction zone, while additional ion optics are used to create focused ion beams or confine ions to a certain region. In other applications, small gradients associated with presheaths can be used to deflect ions of very high energy, using the so-called electrostatic plasma lens effect. ${ }^{6}$ Most of the low pressure plasma sources developed so far provide plasma densities, $n_{\mathrm{o}}$, in a range from $10^{13}$ to $10^{18} \mathrm{~m}^{-3}$ with ion energies from tens of $\mathrm{eV}$ up to $\mathrm{keV}$ to induce surface modifications. This gives a span from microns up to tens of $\mathrm{cm}$ for the sheath thickness. Despite this broad range of size, the sheath diagnostics and also the theoretical coverage are still limited. While low electrical field measurements with good spatial resolution are very challenging, ${ }^{7-9}$ the theoretical investigation has mathematical difficulties to describe the sheath and decide the sheath edge in a model that can take into account the plasma, the presheath, and the sheath in the same set of equations. ${ }^{10-12}$ Having already this complexity, little attention was paid to two- or three-dimensional aspects of the sheath. ${ }^{13,14}$ However, the recent discovery of discrete and modal focusing effects, including progress in numerical capabilities to solve three-dimensional potential structures in

\footnotetext{
${ }^{a)}$ E-mail: eust@dtu.dk

${ }^{b)}$ Deceased.
}

plasma, has changed this situation significantly. ${ }^{15-17}$ For example, instead of scanning an asymptotic potential distribution over less than $1 \mathrm{~cm}$ distance with complex laser diagnostics for detecting the local electric field, one can measure the impact radius, $r_{\text {imp }}$, associated with the discrete focusing, as a quantity directly related to the sheath thickness. This approach can be used both for sheaths created by positive or negative ions. ${ }^{15}$ Moreover, the existence of the modal focusing effect makes it possible to capture very small details of the sheath response to magnetic field or multi-ion species plasmas. The basic phenomenology and the main applications developed so far have been reviewed recently. ${ }^{18}$

The aim of this work is to bring additional details on the fine structure of the modal focusing effect that can be used to strengthen the capability of a plasma-sheath-lens to be used as a plasma-sheath-surface monitoring system.

A two-dimensional cross section of the potential distribution for a conducting disk electrode $\left(5 \mathrm{~mm}\right.$ in radius, $R_{\mathrm{o}}$, insulated on the back) calculated by using the finite element method described in detail elsewhere ${ }^{15,16,18}$ is presented in Fig. 1. The simulation parameters are $n_{\mathrm{o}}=10^{15} \mathrm{~m}^{-3}$, electron temperature, $T_{\mathrm{e}}=2.2 \mathrm{eV}$, electrode potential, $V_{\mathrm{o}}=-150 \mathrm{~V}$, plasma potential, $V_{\mathrm{pl}}=0 \mathrm{~V}$, floating potential $V_{\mathrm{f}}=-7 \mathrm{~V}$, and $\mathrm{Ar}$ ion mass. Ions move under the influence of the electrostatic force, with mass independent trajectories and different time of flight for different masses. The secondary electron emission at the probe surface is neglected. According to the Bohm criterion, the sheath edge, $V_{\mathrm{sh}}$, should be $-1.1 \mathrm{~V}$. However, in order to avoid a too close proximity with respect to the integration boundary, a sheath edge, $\Sigma$, of $V_{\text {sh }}=-5 \mathrm{~V}$ (slightly higher than $V_{\mathrm{f}}$ ) is used as the start location to inject ions, with initial velocities given by this potential difference with respect to plasma $\left(V_{\mathrm{pl}}=0 \mathrm{~V}\right)$, and then calculate their trajectories until they reach the electrode surface. Some relevant trajectories are emphasized with capital letters at entrance locations on $\Sigma$ and with corresponding 


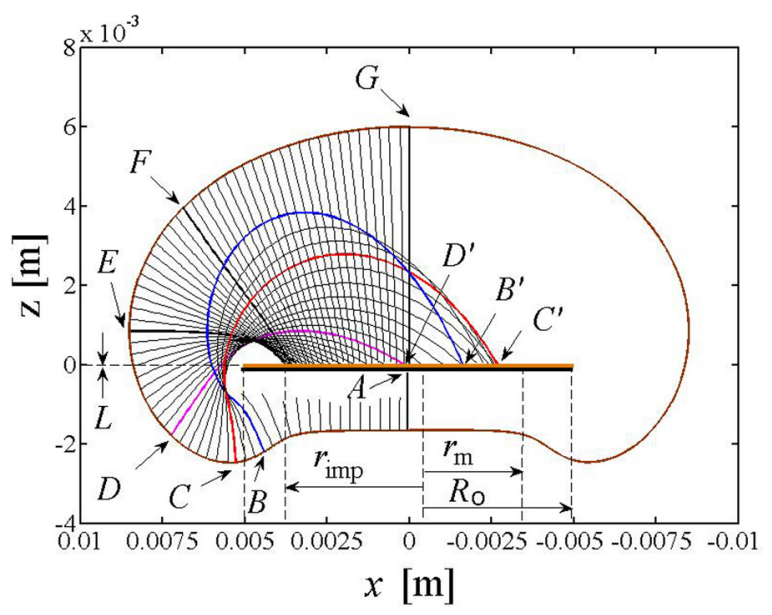

FIG. 1. Two-dimensional cross section of the potential distribution and individual ion trajectories for $n_{\mathrm{o}}=10^{15} \mathrm{~m}^{-3}, T_{\mathrm{e}}=2.2 \mathrm{eV}, V_{\mathrm{o}}=-150 \mathrm{~V}, V_{\mathrm{pl}}=0$, and $V_{\mathrm{sh}}=-5 \mathrm{~V}$.

prime letters at the electrode surface. Ions entering the sheath from $A$ to $B$ do not reach the electrode surface and are collected on the back of the electrode, charged at floating potential. $B$ is the first trajectory reaching the electrode at $B^{\prime}$ and $C$ gives the longest flight over the surface reaching location $C^{\prime}$. $D$ reaches the center of the electrode at $D^{\prime}$, while all trajectories from $E$ to $F$ are focused to a very narrow part of the electrode surface. $G$ is the only trajectory reaching the surface at normal incidence. Most aspects of this behavior were recently reviewed, including the definitions of passive surface, the discrete focusing effect, and impact radius, $r_{\text {imp }}$, so we will only briefly mention that the passive surface is formed at the disk edge and is associated with no ion impact (see the surface corresponding to $r_{\mathrm{imp}}<x<R_{\mathrm{o}}$ ). Ions from the large collection area between $E$ and $F$ form the discrete focusing effect, ${ }^{15}$ while ions from $B$ to $E$ contribute to the modal focusing effect that can be identified with a modal spot at location $D^{\prime}$ for disk electrodes (due to convergence to a spot of all ions entering from locations adjacent to $D$ ) and modal lines for square electrodes. ${ }^{14}$

Let us now concentrate on location $C^{\prime}$, defined as modal radius, $r_{\mathrm{m}}$, which depends on the sheath edge profile near location $C$ or more generally, on the ions entering the sheath with $z<0$, where $z$ is the coordinate perpendicular to the electrode surface (see location $L$ differentiating the sign of $z$ in Fig. 1). This fact was already demonstrated for $V_{\mathrm{o}}=-300 \mathrm{~V}$ and $V_{\mathrm{sh}}=-1.1 \mathrm{~V}$ and $9 \mathrm{~V}$, predicting that location $C^{\prime}$ can in fact reach the electrode reducing the passive surface, with $r_{\text {imp }}<r_{\mathrm{m}}<R_{\mathrm{O}}$ while $V_{\mathrm{o}}=-150 \mathrm{~V}$ will predict a $r_{\mathrm{m}}<r_{\text {imp. }}{ }^{17}$ If confirmed by experiments, this will give one the possibility to investigate the sheath edge and thickness by monitoring $r_{\mathrm{m}}$ in parallel with simulations of ion kinetics within the sheath. By uniformly distributing 63812 ions over the 3D sheath edge $\Sigma$ simulated in Fig. 1, we could separate them, using the $z=0$ plane, in 26114 ions with $\mathrm{z}<0$ and 37698 ions with $\mathrm{z} \geq 0$. The ion impact locations on the electrodes surface are presented in Fig. 2(a) for $z<0$, (b) for $\mathrm{z} \geq 0$, and (c) for all $z$, while (d) presents the averaged incidence angle on the surface. $L_{1}$ is the result of $z$ separation (positive or negative), $F_{1}$ denotes the modal focusing location linked to $r_{\mathrm{m}}$, and $D_{1}$ is the discrete focusing location linked to $r_{\text {imp. A similar }}$ simulation but for $V_{\mathrm{o}}=-300 \mathrm{~V}\left(n_{\mathrm{o}}=10^{15} \mathrm{~m}^{-3}, T_{\mathrm{e}}=2.2 \mathrm{eV}\right)$
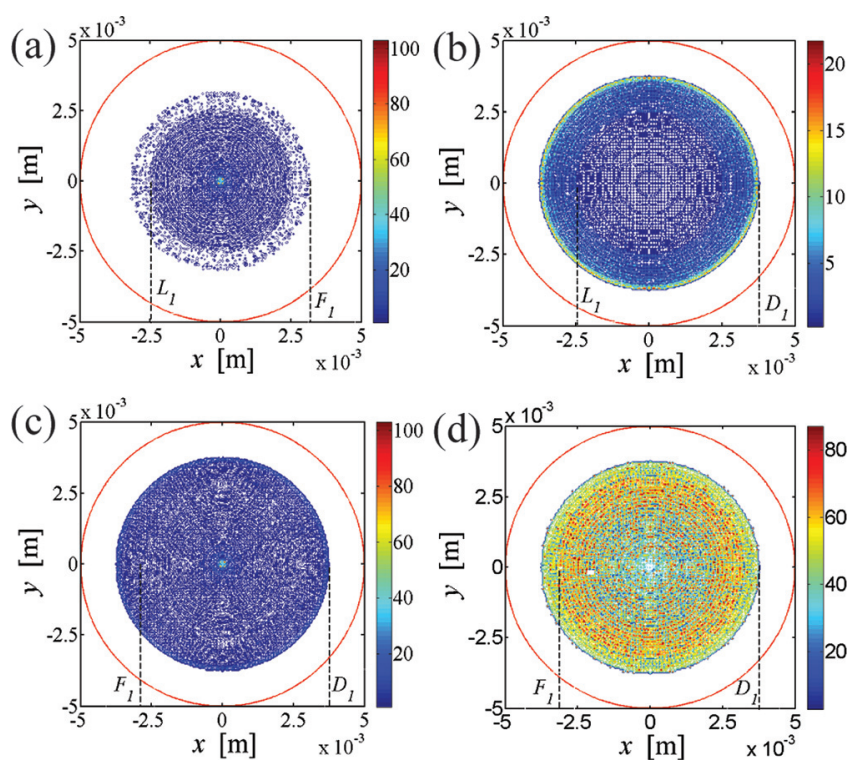

FIG. 2. Ion impact locations on the electrodes surface for $V_{\mathrm{o}}=-150 \mathrm{~V}$ ( $n_{\mathrm{o}}=10^{15} \mathrm{~m}^{-3}$ and $T_{\mathrm{e}}=2.2 \mathrm{eV}$ ) with (a) $\mathrm{z}<0$ (26114 ions), (b) for $\mathrm{z} \geq 0$ (37698 ions), and (c) for all $z$, while (d) presents the averaged incidence angle on the surface. $L_{1}$ is the result of $z$ separation. $F_{1}$ denotes the modal focusing location linked to $r_{\mathrm{m}}$ and $D_{1}$ the discrete focusing location linked to $r_{\text {imp. }}$

with (a) 23470 ions for $z<0$, (b) 41108 ion for $z \geq 0$, (c) all ions and (d) the incidence angle, is presented in Fig. 3, where $L_{2}$ is the result of $z$ separation, $F_{2}$ corresponds to $r_{\mathrm{m}}$ and $D_{2}$ to $r_{\mathrm{imp}}$. Now the presence of the modal spot $(x=0, z=0)$ is very well visible in both (a) and (c) and one can see the shift of $r_{\mathrm{m}}$ and $r_{\text {imp }}$ due to a thicker sheath. The incident angle is expected to have an impact on the etching yield at the surface and can be correlated with the ion dose as to calculate the depth profile after etching.

In order to test the simulation results, $0.5 \mathrm{~mm}$ thick silicon disks of $R_{\mathrm{o}}=5 \mathrm{~mm}$, insulated on the back with alumina disks of similar dimensions, were exposed in plasma $\mathrm{Ar} / \mathrm{SF}_{6}$
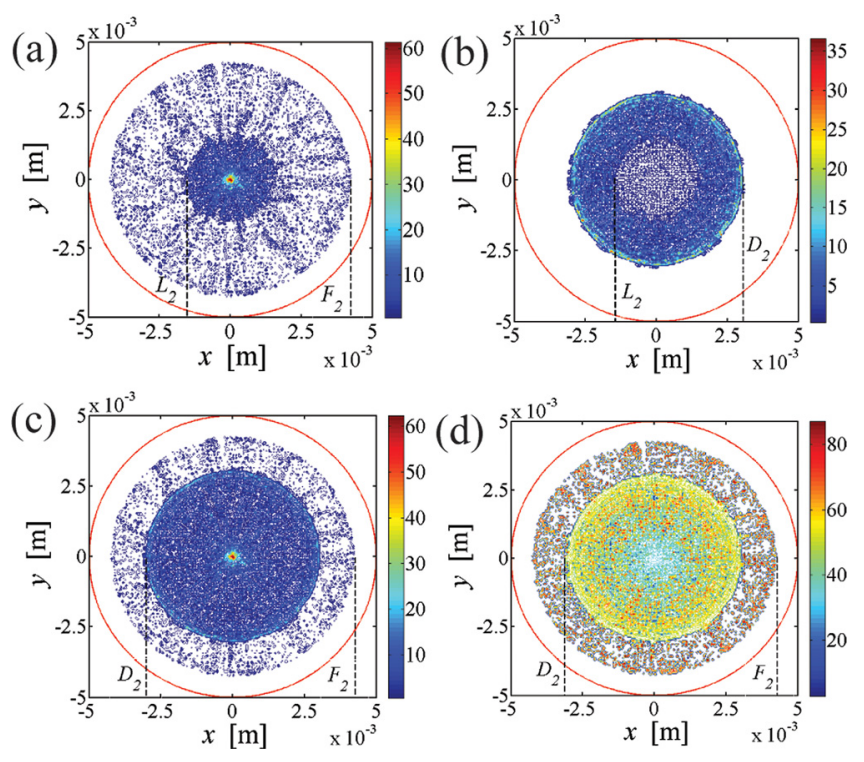

FIG. 3. Ion impact locations on the electrodes surface for $V_{\mathrm{o}}=-300 \mathrm{~V}$ $\left(n_{\mathrm{o}}=10^{15} \mathrm{~m}^{-3}, T_{\mathrm{e}}=2.2 \mathrm{eV}\right)$ with (a) 23470 ions for $z<0$, (b) 41108 ion for $z \geq 0$, (c) all ions and (d) the incidence angle. $L_{2}$ is the result of $z$ separation, $F_{2}$ corresponds to $r_{\mathrm{m}}$ and $D_{2}$ to $r_{\mathrm{imp}}$. 
(Ar: $2 \mathrm{sscm}$ and $\mathrm{SF}_{6}: 0.3 \mathrm{sccm}$ ) at $3 \mathrm{mTorr}$, for $t=15 \mathrm{~min}$, using a magnetic multipolar-confined plasma source presented in detail elsewhere ${ }^{19}$ for a discharge current of $100 \mathrm{~mA}$ and discharge voltage of $70 \mathrm{~V}$. The shaft supporting the disks was a thin ceramic tube of $0.6 \mathrm{~mm}$ in diameter and $50 \mathrm{~mm}$ in length and no noticeable influence of it in the current density distribution over the electrode surface was observed in simulations or experiments. ${ }^{15,16}$ The surface profile after etching by incident ions is presented in Fig. 4 for (a) $V_{\mathrm{o}}=-150 \mathrm{~V}$ and (b) $-300 \mathrm{~V}$, respectively. The depth profile, after compensating for the bowing effects resulting from phase shift interferometry, is presented in (b) for (a) and in (d) for (c). Besides the already known presence of the discrete ion focusing and of the modal spot, one more ring can be observed in each sample. For easier identification, the onedimensional depth profiles for the two samples from Fig. 4 are presented in Fig. 5. The locations corresponding to discrete focusing are indicated with $D_{1}$ and $D_{2}$, those of the modal spot with $M_{1}$ and $M_{2}$ while the additional rings with $F_{1}$ and $F_{2}$, where indexes 1 and 2 are used to differentiate between (b) and (d) in Fig. 4, respectively. By correlation with Fig. $1, F_{1}$ corresponds to $C^{\prime}$ and is the result of the turning point in the scanning of the electrode surface by ion trajectories entering the sheath near $C$. Due to the sharp delineation regarding the ion impact locations, in a similar way with discrete focusing, the etching profile results in a distinct and easily measurable $r_{\mathrm{m}}$ of $2.28 \mathrm{~mm}\left(<r_{\mathrm{imp}}=3.77 \mathrm{~mm}\right)$ for $V_{\mathrm{o}}=-150 \mathrm{~V}$ and $r_{\mathrm{m}}=4.21 \mathrm{~mm}\left(>r_{\mathrm{imp}}=3.51 \mathrm{~mm}\right)$ for $V_{\mathrm{o}}=-300 \mathrm{~V}$. The sheath front at the electrode center $(x=y=0)$ for $V_{\mathrm{sh}}=-1.1 \mathrm{~V}$ has been estimated from simulations at $z=6.15 \mathrm{~mm}$ for $V_{\mathrm{o}}=-150 \mathrm{~V}$ and $z=7.63 \mathrm{~mm}$ for
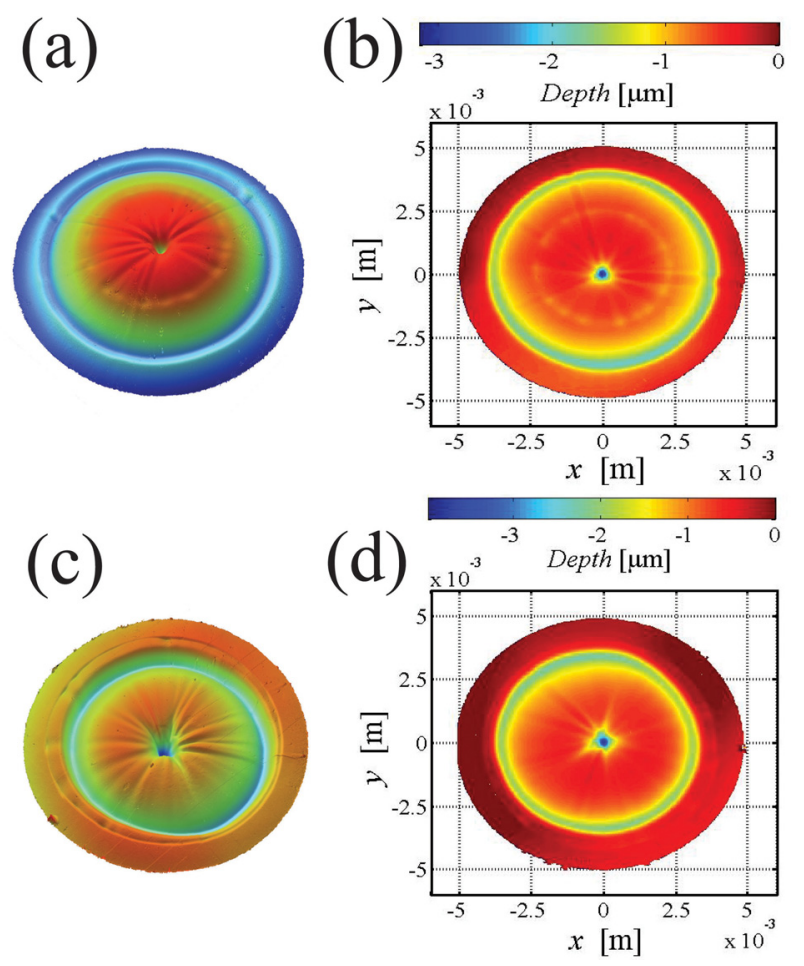

FIG. 4. The surface profile after etching by impinging ions for (a) $V_{\mathrm{o}}=-150 \mathrm{~V}$ and (b) $-300 \mathrm{~V}$ in $\mathrm{Ar} / \mathrm{SF}_{6}$ at $3 \mathrm{mTorr}$, for a discharge current of $100 \mathrm{~mA}$, discharge voltage of $70 \mathrm{~V}$, and $15 \mathrm{~min}$ exposure time. The depth profile is presented in (b) for (a) and in (d) for (c).

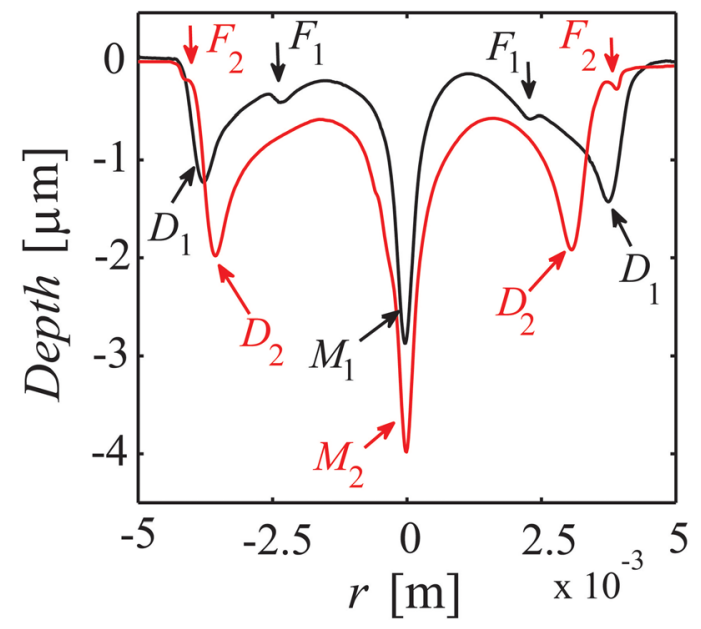

FIG. 5. One-dimensional depth profiles for the two samples presented in Fig. 4. Locations corresponding to discrete focusing are indicated with $D_{1}$ and $D_{2}$, those of the modal spot with $M_{1}$ and $M_{2}$ and the additional rings with $F_{1}$ and $F_{2}$. Indexes 1 and 2 are used to differentiate between (b) and (d) in Fig. 4.

$V_{\mathrm{o}}=-300 \mathrm{~V}$, respectively. This change in sheath thickness of $1.48 \mathrm{~mm}$ resulted in an averaged shift for discrete focusing of $0.39 \mathrm{~mm}$ and of $1.64 \mathrm{~mm}$ for the modal ring. It proves that the modal ring is about four times more sensitive to small changes in the sheath thickness.

It was shown recently that a small electrode can be biased positively up a few hundred volts without affecting the plasma potential in an electronegative discharge as long as the negative ion to electron density ratio, $n_{\mathrm{ni}} / n_{\mathrm{e}}$, is higher than about $20{ }^{18,19}$ For this situation, the sheath is mainly formed by negative ions that are following the same kinetics as presented for positive ions, exhibiting the discrete and modal focusing effects. ${ }^{15}$ Despite an increasing interest in surface processing under low-charging effect by negative ion beams ${ }^{20}$ the negative ion sheath has been investigated to a much lower extent. The positive and negative ion species are always different in complex reactive gases used for etching, such as $\mathrm{CF}_{4}, \mathrm{C}_{4} \mathrm{~F}_{8}$, and $\mathrm{SF}_{6}$. This situation raises a basic question regarding the sheath thickness of positive or negative charges for the same applied bias with respect to $V_{\mathrm{pl}}$. Several attempts have been made in this regard ${ }^{8}$ but yet a questions-free experiment, able to clearly compare the thicknesses of the two sheaths, has not been reported.

The depth profiles for two disks exposed for $15 \mathrm{~min}$ to (a) $-300 \mathrm{~V}$ and (b) $300 \mathrm{~V}$ with respect to plasma potential are presented in Fig. 6, where $V_{\mathrm{pl}}=-11 \mathrm{~V}, n_{\mathrm{o}}=1.2 \times 10^{15} \mathrm{~m}^{-3}$, $T_{\mathrm{e}}=1.7 \mathrm{eV}$, and $n_{\mathrm{ni}} / n_{\mathrm{e}}=57$. The disks were mounted on the same shaft and plasma parameters were monitored with an additional probe making sure that none of the parameters have changed during the 30 min experiment by more than $2 \%$. The diagonal scans of the depth profiles are presented in Fig. 6(c) with the full line representing positive ions and the dashed line representing negative ions. This gives an impact radius for positive ions $\left(r_{\mathrm{pi}}\right)$ of $3.2 \mathrm{~mm}$ and only $2.2 \mathrm{~mm}$ for negative ions $\left(r_{\mathrm{ni}}\right)$, a result that clearly concludes a considerably thicker sheath by negative ions. The modal ring formed by positive ions indicated with $K_{1}$ and $K_{2}$ is well visible, while it could not be identified for negative ions. One explanation can be that the location $C^{\prime}$ (see Fig. 1) exceeded $R_{\text {o. }}$. 

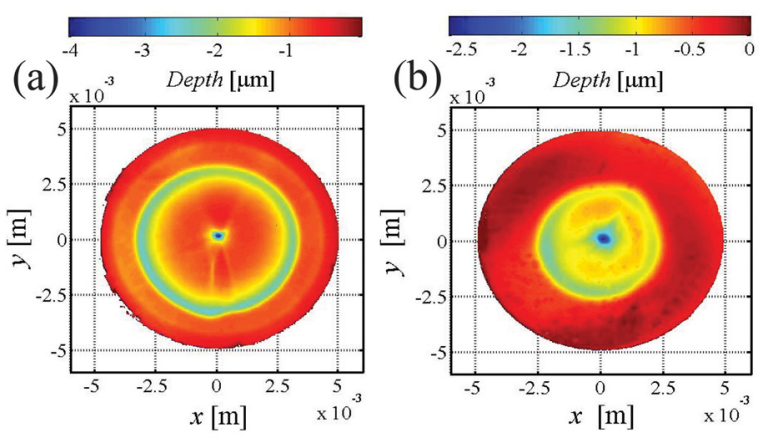

(c)

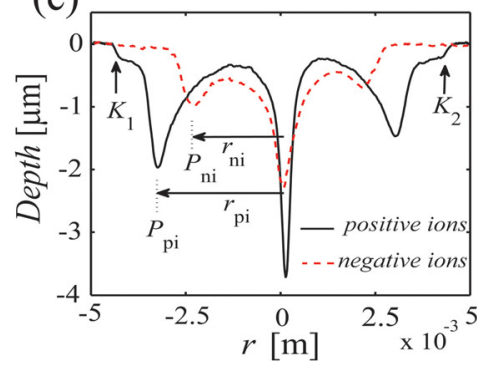

FIG. 6. The depth profiles for two disks exposed for $15 \mathrm{~min}$ to (a) -300 $\mathrm{V}$ and (b) $300 \mathrm{~V}$, where $V_{\mathrm{pl}}=-11 \mathrm{~V}$, $n_{\mathrm{o}}=1.2 \times 10^{15} \mathrm{~m}^{-3}, T_{\mathrm{e}}=1.7 \mathrm{eV}$, and $n_{\mathrm{ni}} / n_{\mathrm{e}}=57$. The diagonal scans are presented in (c) with full line for positive ions and dashed line for negative ions.
In conclusion, a modal ring has been identified in relation with ion focusing by a three-dimensional plasmasheath-lens formed by disk electrodes. The modal ring was formed by the ions entering the sheath at locations facing the back of the electrode and was proven to be about four times more sensitive than the discrete focusing to changes in the sheath thickness. Depending on plasma parameters, electrode dimensions, and applied bias, the modal ring can be located at a certain location that can be predicted by simulations. This property can be used to monitor small variations in the sheath structure with direct application on plasma diagnostics and basic investigations of sheath.

This work was supported by a mobility grant of E.S. at Venture Business Laboratory, Nagoya University, Japan and by internal founding at Technical University of Denmark and is dedicated to the very premature loss by illness of a collaborator and friend, Associate Professor Masahito Yamaguchi, who contributed to this research in the initial stage.
${ }^{1}$ N. Hershkowitz, Phys. Plasmas 12, 055502 (2005).

${ }^{2}$ H. Abe, M. Yoneda, and N. Fujiwara, Jpn. J. Appl. Phys., Part 147, 1435 (2008).

${ }^{3}$ J. G. Han, J. Phys D: Appl. Phys. 42, 043001 (2009).

${ }^{4}$ H. Neumann, M. Tartz, F. Scholze, T. Chasse, H. Kersten, and H. Leiter, Contrib. Plasma Phys. 47, 487 (2007).

${ }^{5}$ J. Pelletier and A. Anders, IEEE Trans. Plasma Sci. 33, 1944 (2005).

${ }^{6}$ Yu. Chekh, A. Goncharov, I. Protsenko, and I. G. Brown, Appl. Phys. Lett. 86, 041502 (2005).

${ }^{7}$ U. Czarnetzki, D. Luggenholscher, and H. F. Dobele, Plasma Phys. Sci. Technol. 8, 230 (1999).

${ }^{8}$ K. Takizawa, A. Kono, and K. Sasaki, Appl. Phys. Lett. 90, 011503 (2007).

${ }^{9}$ E. Barnat and G. A. Hebner, J. Appl. Phys. 101, 013306 (2007).

${ }^{10}$ K. U. Riemann, Plasma Sources Sci. Technol. 18, 014007 (2009).

${ }^{11}$ R. N. Franklin, J. Phys. D: Appl. Phys. 37, 1342 (2004).

${ }^{12}$ N. Sternberg and V. Godyak, IEEE Trans. Plasma Sci. 31, 665 (2003).

${ }^{13}$ T. E. Sheridan, Phys. Plasmas 7, 3084 (2000).

${ }^{14}$ E. Stamate and K. Ohe, Appl. Phys. Lett. 78, 153 (2001).

${ }^{15}$ E. Stamate and H. Sugai, Phys. Rev. Lett. 94, 125004 (2005).

${ }^{16}$ E. Stamate and H. Sugai, Phys. Rev. E 72, 036407 (2005).

${ }^{17}$ E. Stamate, N. Holtzer, and H. Sugai, Appl. Phys. Lett. 86, 261501 (2005).

${ }^{18}$ E. Stamate, Plasma Phys. Controlled Fusion 54, 124048 (2012).

${ }^{19}$ M. Draghici and E. Stamate, J. Phys. D: Appl. Phys. 43, 155205 (2010).

${ }^{20}$ Y. I. Jhon, K. S. Min, G. Y. Yeom, and Y. M. Jhon, Appl. Phys. Lett. 105, 093104 (2014). 